\title{
P2P business and legal models for increasing accessibility to popular culture
}

\author{
Eitan Altman ${ }^{1}$, Sulan Wong ${ }^{2}$, and Julio Rojas-Mora ${ }^{3}$ \\ 1 INRIA Sophia Antipolis, 2004 route des Lucioles, 06902 Sophia Antipolis, France. \\ eitan.altman@sophia.inria.fr \\ 2 University of A Coruña, Faculty of Law, Campus Elviña, 15071 A Coruña, Spain. \\ swong@udc.es \\ 3 LIA, University of Avignon, 339 Ch. des Meinajaries, Agroparc BP 1228, 84911 \\ Avignon Cedex 9, France. julio.rojas@univ-avignon.fr
}

\begin{abstract}
Recent publications have reached conflicting conclusions on wheather allowing users to have unlimitted free access for downloading music and films is benefitial or not for the content provider industrty. Not only do models differ in their conclusions, but there has also not been an agreement on the validity of data on the impact of free access to music and films using P2P on the profits of CPIs and on the demand for CDs and DVDs. We provide in this paper a model that allows to study this question using elementary mathematical tools. In particular, it includes the effect of sampling on the the willingness to buy. Preliminary numerical experiments show that benefits are maximized by avoiding any control measures against unauthorized sampling over P2P networks.
\end{abstract}

\section{Introduction}

Fast evolution of the P2P technology in last years has increased the wide spread access to popular culture (music and films). It has promoted file sharing at the expense of free riding. This evolution created a major conflict between internautes and content provider industry. While a legal battle is being held in courts and in the legislation area, the conflict also finds its echoes in scientific analysis of experimental data and of the economy of P2P networks. In particular, some publications conclude that the music and film industry gains from $\mathrm{P} 2 \mathrm{P}$ where as other conclude the contrary. The gains can be attributed to a sampling effect: by downloading music, a customer can get more information that may increase its willingness to purchase a hard copy of the CD. On the other hand, the availability of free copies for download considerably decreases the demand for costly electronic copies sold by the companies and may constitute for many users a satisfactory alternative to the original CD thus decreasing again the incomes of the content providers industry ${ }^{1}$.

\footnotetext{
${ }^{1}$ According to the 2009 IFPI [1], by 2008 its 3.7 billion dollars digital music business model had, on an international level, a $25 \%$ estimated growth. The RIAA [2] reported that sales of digital music have grown in 2008 by $30 \%$, which represents 1.6 billion dollars, and constituted $32 \%$ (shipments) of the total market value and 2.7 billion dollars in total sales. The market for physical recorded music have fallen $28 \%$ to 5.8 billion dollars.
} 
The aim of this paper is first to propose an economic model that predicts the impact of the P2P networks on the access to culture on the one hand, and on the incomes of the content providers industry (CPI) on the other. In particular we aim at understanding the impact of actions of the CPI on these two performance measures. Based on the economic model, we identify numerically conditions for win-win business models in which CPIs can see their benefit increase while increasing the accessibility to music and films.

We consider the following two categories of actions that can be taken by the CPI:

1. Sampling: Making contents available for download at their own site at a cost that they can determine.

2. Measures against unauthorized downloads: Investment in fighting (e.g. in courts) against non-authorized downloads.

We provide in the next section an overview of the economic aspects of sampling and of legal measures againsnt unauthorized downloads. We then introduce an economic model and compute the expected income of CPI taking into account the impact of their actions on the availability of the files at the P2P networks, on the demand for sampling of the file and for purchasing it.

\section{Sampling and Legagl Measures against Downloads}

Thanks to new technologies such as P2P networks, consumers have found tools that allow them to freely explore, choose, listen and select what music to purchase. Sampling has escaped from the monopolistic control of the music producer as an advertisement strategy, and has positioned itself in the hands of consumers. We provide below a sohrt overview on the legal and economic aspects of sampling.

\subsection{The discussion of sampling at American jurisprudence}

After the RIAA tried to sue more than 30,000 individual file sharers since 2003 [3], the CPI has changed judicial harassment for a concerted strategy with Government and ISPs that, by using "cease and desist" letters, seeks to discourage file sharing with the threat of Internet service suspension. Still, we will review the two main legal cases in American jurisprudence that have looked at the subject of sampling. In A\&M Records et al. v Napster [4], the defendant argued that its users, in a behavior that resembled fair use, sampled several musical products to make a decision on what to purchase. The Court concluded that sampling is commercial in nature, can not be done by consumers without the producer's authorization, and, hence, can not be identified as fair use. Napster, based on [5], even appealed on the positive effects that sampling creates on the market. The Court of Appeals again agreed with the plaintiffs, for whom file-sharing not only hurts CD sales, but also blocks the new markets which the copyright owners could and are entitled to create. Moreover, the Court of Appeals said that although sampling might have a positive effect on the market in general, the increase in sales does not deprive the owners of the right to license their works.

Five years later, the argument about the effect of sampling is reviewed in in BMG Music, et al. v. Cecilia Gonzalez [6], who was sued for 30 songs downloaded from P2P platforms. Gonzalez argued that she could not have infringed 
copyright, because her downloading activity was just a previous step before purchasing music. The Court, in line with the Napster ruling, denied any identity of the sampling with the doctrine of fair use and found in the Grokster ruling [7] empirical support that led it to conclude that downloaded music "is a close substitute for purchased music"

\subsection{Trial costs and attorney's fees}

RIAA, in its legal strategy, presents the defendants a pre-litigation extra-judicial settlement that ranges between US $\$ 3,000$ and 5,000. People who accept the offer are not brought to trial. A conviction in trial would mean between US\$ 750 [8, $\S 504$ (c) (1)] and US\$ $150,000[8, \S 504$ (c) (2)] in statutory damages per musical work infringed. However, any civil action $[8,17 \$ 505]$ on copyright can lead to the recovery of litigation costs by any party involved in the process and the court may fix attorneys' fees to be recovered as part of the costs. On 2007, the United States District Court for the Western District of Oklahoma [9] ordered the plaintiff to pay Debbie and Amanda Foster US\$ 61,576.50 as attorney's fees, and close to US\$ 7,000 for litigation costs. On 2008, Tanya Andersen was awarded US\$103,175 in Attorney's fees and US\$ 4,659 in costs, by the United States District Court for the District of Oregon [10].

\subsection{Effects of sampling on sales: theoretical and empirical results}

Experience goods [11] identify assets that need to be consumed before knowing their satisfaction level. Consumers make an initial selection based on information from indirect sources and will continue testing until the cost of a new trial exceeds the expected growth in satisfaction. Peitz and Waelbroeck [12], assuming that music is an experience good, argue that P2P networks offer the possibility of sampling in music. In their basic model the benefits obtained by the CPI can be increased due to a more informed consumer which would be willing to pay more for albums he really wants to buy. In an extended model, profits will grow even with lower prices of the albums, thanks to savings the CPI would have in costs of marketing and promotion.

Liebowitz $[13,14]$ opposes this position and, based on data collected by the CPI on sales of CDs, argues that the growth of file sharing through P2P networks is the cause of the fall in per capita album sales. To him, sampling simply gives the consumer a more accurate view of his musical taste, but it does not increase his music buying level[15]. Countering RIAA's argument, Liebowitz believes that downloading an album can not be correlated with one or more unsold albums, and a replacement rate of about 5 to 6 CD's downloaded per each unsold CD is more realistic. Analysis made on surveys using different methodologies agree that sampling hurts music sales. Rob and Waldfogel [16], interviewed college students in the United States, finding this impact close to $9 \%$. Michel [17] and Hong [18], based on data from the Consumer Expenditure Survey (CEX), found a drop in sales of $13 \%$ and $7.6 \%$ respectively. Using data from the European Consumer Technographics survey, Zentner [19] concludes that file-sharing reduces the probability of buying music by $30 \%$, and music sales by $7.8 \%$.

On the other side, using data from German college students, Oberholzer-Gee and Strumpf [20] could not find a direct relationship between file-sharing and declining sales of CD's, obtaining an effect "statistically indistinguishable from 
zero". A more recent study [21] commissioned by the Dutch government, argues that even when buying and file-sharing sometimes go hand in hand, they are not mutually exclusive, i.e., there is no direct relationship between downloading files protected by copyright and purchasing music in physical format . One of the findings points that file sharers are not more or less willing to buy music that other people, and those file sharers that buy music do not buy more or less music than non file sharers, but they acquire more value added products, like concert tickets and promotional items (licensing).

Andersen and Frenz [22], analyzing microdata from the Canadian population as a whole, concluded that there is no clear evidence to say that file sharing affects music sales. However, the group that shares files has a clear positive relationship between the number of downloads per month and music CD's buying, of about 0.44 albums bought for each album this group downloads . Furthermore, they observe a negative indirect influence of album prices in album sales. Price, along assortment of authors, performers and genres (even those not available in traditional stores), and desire to discover new music, is also the main factor that pushes the consumer to download music instead of buying CD's, according to Sanduly [23], who in a study based on the Spanish music market, finds a "strong heterogeneity" in the relationship between the consumption of music via P2P networks and the consumption of music through physical format (CD's).

\section{$3 \quad$ P2P business model for the CPI}

Define $U$ to be the set of policies available for the CPI for impacting the demand. A policy $\underline{u}$ has the following components:

1. $u^{(1)}$ is the price per sampling an item at the site of the CPI.

2. $u^{(2)}$ is the cost payed by the CPI for legal and other measures to dissuade sampling at $\mathrm{P} 2 \mathrm{P}$ networks.

Let $a(u)$, the availability of a file in a P2P network, have the following linearized form ${ }^{2}$ :

$$
a(u)=a(0)+\alpha \cdot u^{(1)}-\beta \cdot u^{(2)} ; a(u) \in[0,1]
$$

The initial availability of a file increases with the price charged by the CPI, since higher prices make the use of $\mathrm{P} 2 \mathrm{P}$ a more attractive alternative. On the other hand, it is negatively affected by the investment made by the CPI on control measures. As reported in [22] and [23], price is the most important factor in the decision of making P2P sampling over buying music. Even if the CPI uses an aggressive pricing scheme, the effect of price is still important, so $\alpha \approx \infty$. Conversely, huge investment on control measures has been largely ineffective in reducing availability of music on $\mathrm{P} 2 \mathrm{P}$ networks, so $\beta \approx 0$. For simulation, it is possible to make $\alpha \gg \beta$. This fact was also obtained from analytical consideration in [25], where it was shown that very popular files see their demand decrease by CPI's measures but not their availability.

\footnotetext{
${ }^{2}$ One can use alternative models from [24] for the availability of a file at the P2P network as a function of $u$.
} 


\subsection{Sampling as a branching process}

We propose a dynamic model for the sampling: a sample may change the information of the internaute not only on the particular sampled item but also on other related ones. For example, it may increase or decrease its interest in discovering more products by the same artist. This may lead as a result to new sampling by the internaute. We thus assume that as a result of a sample, a demand for a random number of samples is generated. This means that we model the sampling as a branching process.

Assume that the internaute plans initially to use $X_{1}$ samples. This is a sample of the first generation. Sample $i$ of generation $n$ will cause further creation of $K_{n}^{(i)}$ samples. The total number of extra samples is given recursively by $X_{n}=$ $\sum_{i=1}^{X_{n-1}} K_{n-1}^{(i)}$. Taking expectation and making $\bar{K}(u)_{n-1}=E_{u}\left[K_{n-1}^{(i)}\right]$, we get $E_{u}\left[X_{n}\right]=E_{u}\left[X_{1}\right] \cdot \bar{K}(u)_{n-1}$ We conclude that the total expected number of samples will be: $N=X_{1} /(1-\bar{K}(u))$.

For computation purposes, we shall again use an approximating version of this expression. Using a linear approximation, the expected number of samples $N_{p 2 p}(u)$ at a P2P network and $N_{c p i}(u)$ at the CPI's site, respectively, as a result of a single initial sample would be: $N_{c p i}(u)=N_{c p i}(0)-k^{(1)} u^{(1)}, N_{p 2 p}(u)=$ $N_{p 2 p}(0)-k^{(2)} u^{(2)}$. We found this approximation unsuitable since it does not model the fact that for small value of $u^{(1)}$ and of $u^{(2)}$, we may expect a very high value of $N_{c p i}$ and of $N_{p 2 p}$, respectively. To obtain this, we used the following nonlinear approximations instead:

$$
\begin{aligned}
& N_{c p i}(u)=\min \left(N_{c p i}(0), N_{c p i}(0)-k^{(1)} \cdot \log \left(\delta u^{(1)}\right)\right) \\
& N_{p 2 p}(u)=\min \left(N_{p 2 p}(0), N_{p 2 p}(0)-k^{(2)} \cdot \log \left(\gamma u^{(2)}\right)\right)
\end{aligned}
$$

Next we present a simple first order linear model for the sampling probability. We have:

$$
\underline{p}_{s}(u)=\underline{p}_{s}(0)+C \underline{u}
$$

where $C_{i i} \leq 0$ and $C_{i j} \geq 0, p_{s}^{(1)}(u)$ is the probability of sampling in a $\mathrm{P} 2 \mathrm{P}$ network, and $p_{s}^{(2)}(u)$ is the probability of sampling at the CPI site. Sampling on the CPI site is done by purchasing an electronic version of the song.

We assume that an item is sampled at the CPI's site if it is not available at a P2P network. A successful sample is one in which the requested item was available. The expected amount of successful sampling generated as a result of an initial sampling attempt, at the P2P network and at the the CPI's site, respectively, are given by:

$$
\begin{aligned}
& S_{p 2 p}(u)=p_{s}^{(1)}(u) \cdot a(u) \cdot N_{p 2 p}(u) \cdot d \\
& S_{c p i}(u)=p_{s}^{(2)}(u) \cdot(1-a(u)) \cdot N_{c p i}(u) \cdot d
\end{aligned}
$$

where $d$ is the potential demand for an item. The expected demand for purchase is:

$$
D(u)=\left(1-p_{s}(u)\right) d q_{n}+\left(p_{s}^{(1)}(u) a(u) N_{p 2 p}(u)+p_{s}^{(2)}(u)(1-a(u)) N_{c p i}(u)\right) d q_{s}
$$

where $p_{s}(u)=p_{s}^{(1)}(u)+p_{s}^{(2)}(u)$ is the probability of sampling, $q_{s}$ is the probability that an item is purchased if sampled, and $q_{n}$ is the probability that an item is purchased if not sampled. 


\subsection{The CPI's income}

Sampling generates traffic that benefits to ISPs. We assume that incomes due to subscription are split in a way that a content provider receives an income proportional to the demand it generates. The proportionality coefficient is denoted by $c_{i s p}$. The income of the CPI is thus:

$$
R(u)=\left(u^{(1)}-u^{(2)}+w\right) D(u)+c_{i s p} S_{c p i}(u)
$$

CPI is thus faced with the problem of maximizing $R(u)$ over $u$. $R(u)$ is given by:

$$
\begin{aligned}
R(u)= & \left(u^{(1)}-u^{(2)}+w\right)\left(\left(1-p_{s}(u)\right) d q_{n}+\left(p_{s}^{(1)}(u) a(u) N_{p 2 p}(u)\right)\right) \\
& +\left(u^{(1)}-u^{(2)}+w+c_{i s p}\right) p_{s}^{(2)}(u)(1-a(u)) N_{c p i}(u) d q_{s}
\end{aligned}
$$

where $w$ is the difference between the price of selling an item (hard copy) minus the marginal cost to produce it.

\section{Simulation}

We take for (1) the values $\alpha=0.5$ and $\beta=1.5 \cdot 10^{-9}$. This corresponds to a very popular file for which the measures taken by the service provider have a negligible impact on its availability (see discussion below eq (1)).

Take $u^{(2)}=0.01 *(84,000 * 30,000)=25 \cdot 10^{6}$, obtained from average attorney fees in P2P cases (US\$ 84,000, see end of Section 2.1) and number of cases (around 30,000 [3]). For $u^{(2)}$, according to [21] the actual number of samples per user in P2P networks is difficult to quantify. Still, they use a conservative estimate of under 300 songs downloaded per user in a year, so we will use a value of $N_{p 2 p}(u)=200$. Because the effect from control measures is very small, $N_{p 2 p}(0) \approx N_{p 2 p}(u)$, we will use $N_{p 2 p}(0)=300$. By requesting that (2) coincides with the values at $N_{p 2 p}=200$ and at $N_{p 2 p}=300$, we obtain $k^{(2)}=30$ and $\gamma=10^{-6}$.

From iTunes most know price, we will fix $u^{(1)}=0.99$. For $u^{(1)}$ sampling in CPI sites can be set at $N_{c p i}\left(u^{(1)}=0.99\right)=20$ songs per customer (Apple [26] reports this number per iPod sold). We assume that if the price was zero, users of the CPI site would download at least the same number of songs as in P2P networks, so $N_{c p i}(0)=N_{p 2 p}(0)=300$. This gives $k^{(1)}=7.5$ and $\delta=30$, so $N_{c p i}\left(u^{(1)}=0.99\right) \approx 20$.

With current levels of $u$, let's assume $C_{12} \approx 0$. From [21] we can fix $\mathrm{p}_{s}(u)=$ $(0.7,0.4)$. The best case sampling probability should be close to one for the CPI site and not very far from the actual value for P2P networks, so let's use $\mathrm{p}_{s}(0)=(1.0,0.5)$. To get better regions of $\mathrm{p}_{s}(u) \in[0,1]$, we made $C_{11}=0.61812$, $C_{12}=10^{-9}, C_{21}=0.29391$ and $C_{22}=5.5 \cdot 10^{-9}$.

For 4,5 and 7 we used $d=1$. From [21, 80] we fixed $q_{n}=q_{s}=0.14125$, as the buying behavior for file sharers and non-file sharers is the same. From [27, 360 ] we make $w=3 / 12$ (approx. US $\$ 3$ divided by the average number of songs of a CD). Finally, we fixed $c_{i s p}=0.01$.

but the effect from $u^{(1)}$ is stronger. Demand, shown in Figure 1a grows when both $u^{(1)}$ and $u^{(2)}$ decrease, but when $u^{(2)}$ grows beyond certain threshold, 
Figure1: Contour plots for simulation data.

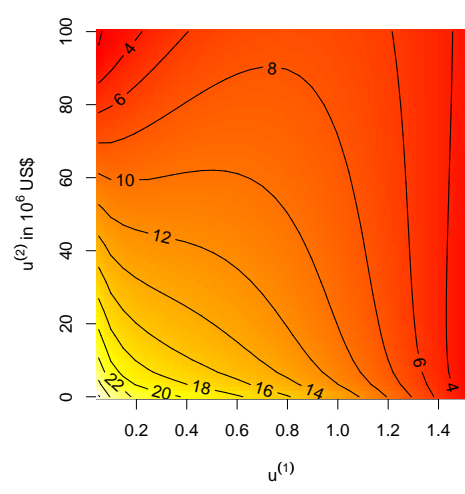

(a) Demand $(D)$.

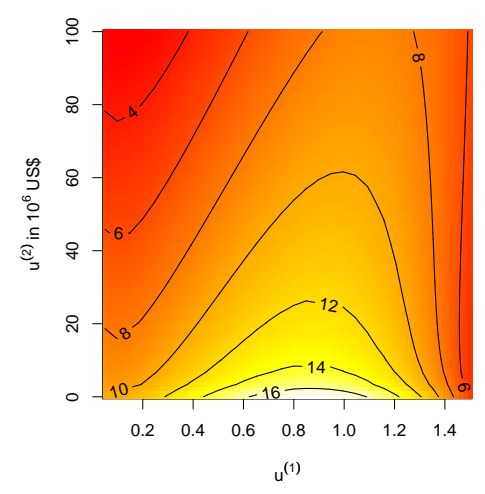

(b) Revenue $(R)$.

demand reaches a maximum level only when the price grows, showing that users increase their use of P2P networks. Revenue finds a maximum level when $u^{(2)}$ is zero and $u^{(1)}$ is between 0.6 and 1.0, as we can see in Figure $1 \mathrm{~b}$ and follows this pattern for any level of $u^{(2)}$. Thus, by avoiding any judiciary control measures against unauthorized sampling over P2P networks, the profits of the CPI are maximized.

\section{Conclusions}

We proposed a modelling approach that takes into account various aspects of sampling that could have an impact on the expected income of the CPI. We studied in particular the impact of various strategies of the CPIs. We used read data to trim the parameters of the simplified model (based mainly on first order approximations). Numerical experiments show that the CPI is better off, in terms of revenues, by abandoning the vast investment in for the prosecution of file sharers.

\section{References}

1. IFPI: Digital Music Report 2009. Technical report, IFPI (2009)

2. RIAA: 2008 Year-End Shipment Statistics (2008)

3. Electronic Frontier Foundation: RIAA v The People: Five years later (2008)

4. United States Court of Appeals for the Ninth Circuit: A\&M Records, Inc., et al. v. Napster, Inc. Nr. 00-16401 (2000)

5. Fader, P.S.: Expert Report. A\&M Records, Inc. et al. v. Napster, Inc. 114 F. Supp. 2d 896 (2000)

6. United States Court of Appeals for the Seventh Circuit: BMG Music, et al., vs Cecilia Gonzalez. No 05-1314 (2005)

7. Supreme Court of the United States of America: Metro-Goldwyn-Mayer Studios et al. v. Grokster, Ltd., et al. No 04-480 (2005) 
8. USC: (United States Code)

9. United States District Court for the Western District of Oklahoma: Capitol Records, Inc., et al., v. Debbie Foster and Amanda Foster No. Civ. 04-1569-W (2007)

10. United States Distric Court, District of Oregon: Atlantic Recording Corporation et al v. Tanya Andersen No. 05-933-AS (2008)

11. Nelson, P.: Information and Consumer Behavior. The Journal of Political Economy 78(2) (1970) 311-329

12. Peitz, M., Waelbroeck, P.: File-Sharing, Sampling and Music Distribution. SSRN eLibrary (2004)

13. Liebowitz, S.J.: Will MP3 downloads annihilate the record industry? The evidence so far. Intellectual property and Entrepreneurship 15 (2004) 229-260

14. Liebowitz, S.J.: Testing File-Sharing's Impact by Examining Record Sales in Cities. SSRN eLibrary (2006)

15. Liebowitz, S.J.: Economists Examine File-Sharing and Music Sales. In Illing, G., Peitz, M., eds.: Industrial Organization and Digital Economy. MIT Press (2006)

16. Rob, R., Waldfogel, J.: Piracy on the high C's: Music downloding, sales displacement, and social welfare in a sample of college students. Journal of Law and Economics XLIX (2006)

17. Michel, N.J.: The Impact of Digital File Sharing on the Music Industry: An Empirical Analysis. Topics in Economic Analysis \& Policy 6(I) (2006)

18. Hong, S.H.: Measuring the Effect of Digital Technology on the Sales of Copyrighted Goods: Evidence from Napster. SSRN eLibrary (2007)

19. Zentner, A.: Measuring the Effect of File Sharing on Music Purchases. The Journal of Law and Economics 49 (2006)

20. Oberholzer-Gee, F., Strumpf, K.: The effect of file sharing on record sales: An empirical analysis. Journal of Political Economy 115(1) (2007)

21. Huygen, A., Rutten, P., Huveneers, S., Limonard, S., Poort, J., Leenheer, J., Janssen, K., van Eijk, N., Helberger, N.: Ups and downs: Economic and cultural effects of files sharing on music, film and games. Technical Report 34782, TNO and SEO and IViR (2009)

22. Andersen, B., Frenz, M.: The Impact of Music Downloads and P2P File-Sharing on the Purchase of Music: A Study for Industry Canada. http://www.ic.gc.ca/ eic/site/ippd-dppi.nsf/eng/h_ip01456.html (2007)

23. Francesco D., S.: CD music purchase behaviour of P2P users. Tecnovation $\mathbf{2 7}$ (2007) $325-334$

24. Gayer, A., Shy, O.: Publishers, artists, and copyright enforcement. Information Economics and Policy 18 (2006) 374-384

25. Wong, S., Altman, E., Ibrahim, M.: P2P Networks: The interplay between legislation and information technology. INRIA Technical Report RR-0689 (2009)

26. Jobs, S.: Thoughts on Music. http://www apple.com/hotnews/thoughtsonmusic/ (2007)

27. Peitz, M., Waelbroeck, P.: An Economist's Guide to Digital Music . CESifo Economic Studies 51(2-3) (2005) 359-428 